\title{
URwaga software as interface for measuring devices
}

\author{
Michat Cupiat ${ }^{1}$, Bogdan Kulig $^{2}$ and Vladimir Pačuta ${ }^{3}$ \\ ${ }^{1}$ University of Agriculture in Krakow, Faculty of Production and Power Engineering, ul. Balicka \\ 116B, 30-149 Krakow, Poland \\ ${ }^{2}$ University of Agriculture in Krakow, Faculty of Agriculture and Economics, Al. Mickiewicza 21, \\ 31-120 Krakow, Poland \\ ${ }^{3}$ Slovak University of Agriculture in Nitra, Department of Crop Production and Grassland \\ Ecosystems, Tr. A. Hlinku 2, 94976 Nitra, Slovak Republic
}

\begin{abstract}
The paper presents computer program URwaga which operates measuring devices at the test stand. The program runs devices that communicate via RS232 interface and devices that are connected to a computer via USB. The obtained data are collected in a cumulative table and then exported to a spreadsheet. The program is applied in the studies on agriculture and mechanical engineering. The application cooperates with factory installations and an original measuring system on Arduino platform.
\end{abstract}

\section{Introduction}

RS-232 interface is a communication bus designed for serial data transmission. The most popular version of this standard enables transfer to the distance not exceeding $15 \mathrm{~m}$ with the maximum speed of $20 \mathrm{~kb} / \mathrm{s}$. Although, the standard was created in the 60's of the 20th century, still there are many devices which use such data transmission. It is particularly related to measuring devices such as e.g. Laboratory scales. Serial ports marked COM1, COM2, COM3 etc. were designed in PC computers.

Old RS232 sockets in modern computers are almost non-existent, they were replaced by USB (Universal Serial Bus). USB's are compliant with the Plug and Play standard and enable easy connection of various devices to a computer [1]. To connect older devices to a computer, it is necessary to use relevant converters. As a result, measuring systems consisting of both modern devices and old generation equipment may be built.

Arduino is an electronic open-source platform based on a micro-controller mounted on the plate with a built-in operation of input/output. The platform is equipped with own programming language. Arduino does not require an external programmer, connection to a computer by means of USB is sufficient. The platform has a household environment with a substantial number of libraries and runs many external devices [2]. Combination of easiness of use with an enormous potential have made those systems extremely popular recently they can be also used in the testing and measuring devices [3] [4].

\footnotetext{
${ }^{1}$ Corresponding author: michal.cupial@urk.edu.pl
} 
PCarrying out scientific research is often related to numerous measurements with various devices. It is mainly related to research areas such as agriculture and horticulture or mechanical engineering [5] [6] [7]. Many times, several measurements are made concerning a single object, e.g., weight, length, width, colour, etc. The obtained results should be entered into a joint data base in a manner that enables clear identification of the object and the measurement number [8]. In case of the test stand consisting of several devices of various producers, data are collected, as a rule, in several locations [9]. Combination of these data in one single base results in the loss of time and may cause errors at aggregation.

The described application must connect several devices in a single measuring system which enables recording of measurements in a strictly defined manner. Although the program was created to operate a specific measuring system, it can be fully configured. It enables development of the stand or creation of new systems. The obtained data are recorded in a file, from where they can be imported to a spreadsheet (MS Excel or LibreOffice).

This paper was financed with a grant from the Ministry for Higher Education for statutory activities

\section{Results}

\subsection{Measuring system}

URwaga software was created for the purpose of the developed measuring stand which consists of four devices. The program stipulates a possibility of connecting another device. A simplified block scheme of the measuring system is presented in figure 1 .

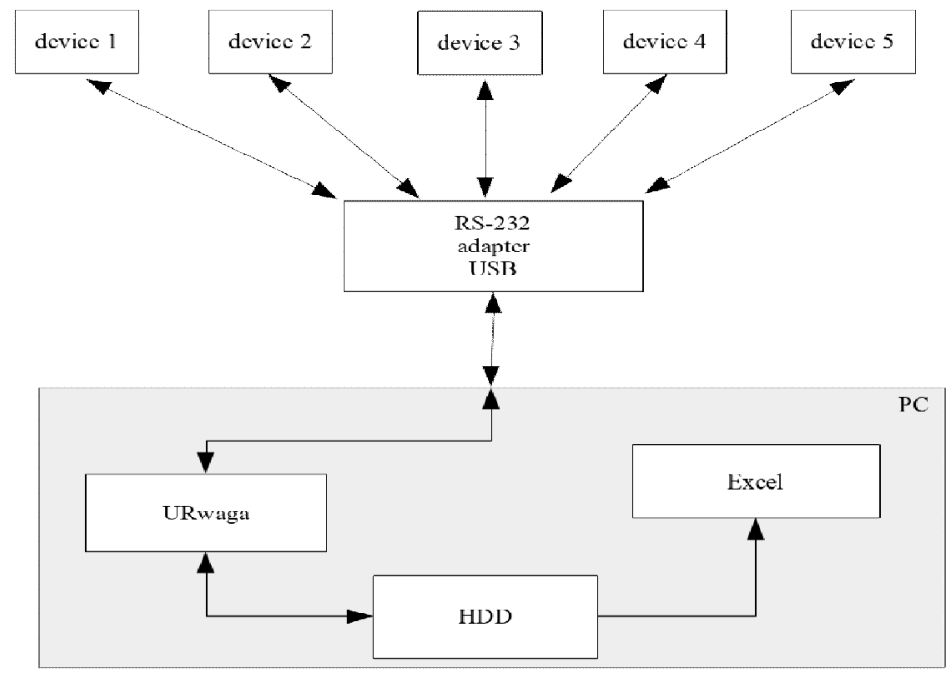

Fig. 1. Block scheme of the measuring system

Weights, an electronic calliper, and devices for measurement of distance/length were connected to the measuring system. An original measuring system built based on Arduino platform was one of the devices. In the measuring system, various devices occur, which generate various measuring signals and operate various parameters of data transmission (e.g. speed). Precision of the measurement of physical values results from the precision of particular devices. Connected laboratory scales required application of a converter (adapter 
RS232/USB), Arduino system could have been connected directly to a USB of the computer. Data after processing in URwaga program were recorded on the disc and then subjected to further treatment in the MS Excel spreadsheet.

\subsection{URwaga computer program}

Figure 2 presents component modules of application. The program communicates through a serial port (COM). A serial port operation module obtains data from the device or sends a control signal to it. A read out from 5 measuring devices, for which relevant COM ports can be ordered and transmission parameters can be defined for each of them, is possible.

The obtained data are then decoded and filtrated. Each device sends data in their own format; thus, it is necessary to leave only numerical data which are a result of measurement. For each device, a way numerical data will be decoded should be defined. A possibility of reading out a "raw" signal from a device was also designed to verify whether the signal is sent through the interface and to define its format.

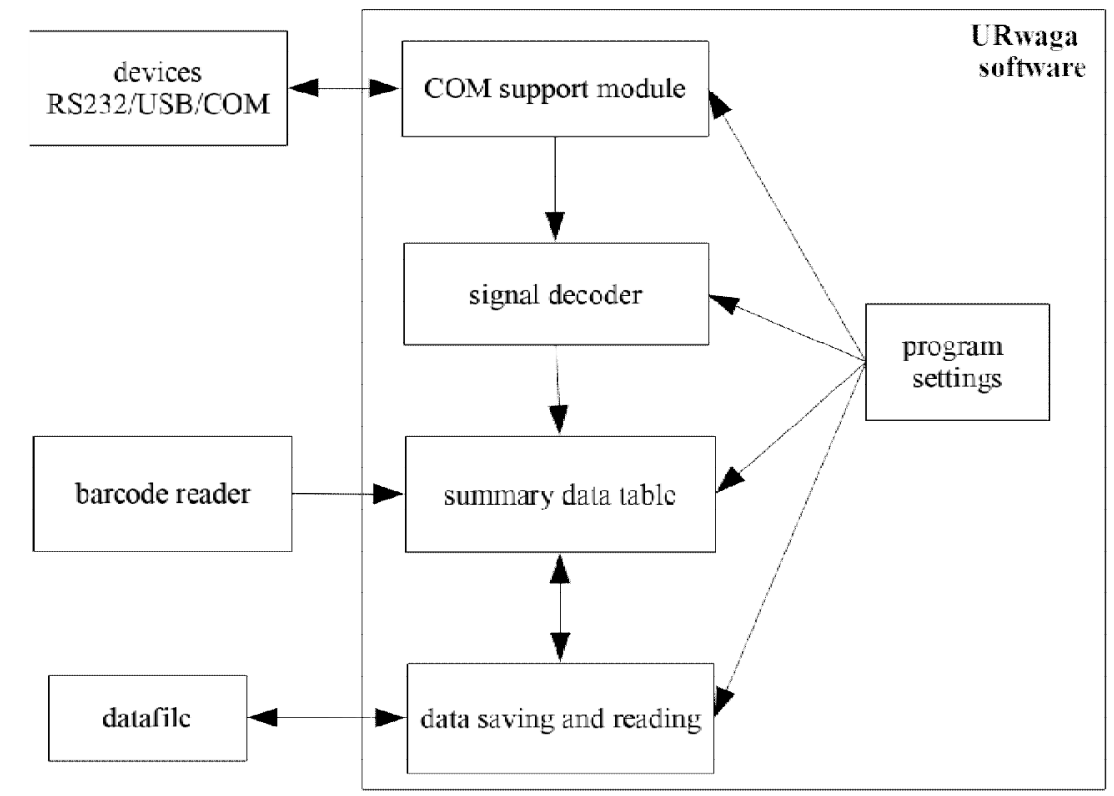

Fig. 2. Block scheme of URwaga program

The read-out values are recorded in the cumulative table. A bar code reader, which reads in an identification number to the table, was designed for identification of an object. Data are collected in a table according to the defined scheme.

Data may be recorded automatically after each measurement or requested by a user. The operation of the measuring stand may be continued after a read out of earlier measurements from a file. The files have cvs format which enables a simple import of data to a spreadsheet.

Settings of COM ports for particular devices, information indispensable for decoding data, file format etc. are recorded in the configuration of the program.

An exemplary screenshot of URwaga program was presented in figure 3 


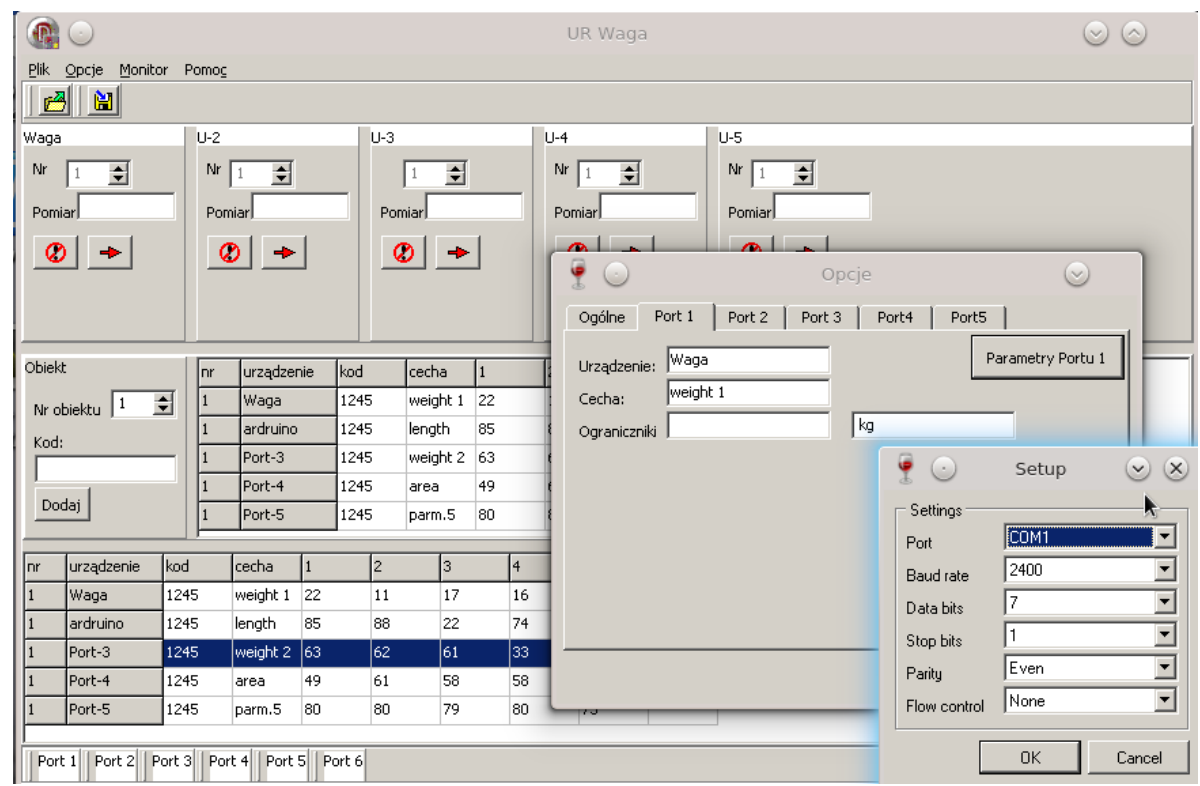

Fig. 3. Screenshot of URwaga software

Outside the measuring window, an open window of configuration and a window with settings of the selected port was showed. The program was written in Delphi, the use of relevant libraries that enable operation of serial ports. Before measurements were started, each port must be open, after the finished cycle of measurements it is closed. Ports are automatically closed when the work with the program is being completed. Signalization of the status of particular communication ports is visible on the status bar and double click of a mouse changes the device status. The program was also protected against errors which may occur during communication with external devices.

\subsection{Measurement system based on Arduino}

One of measuring devices is an original system that uses Arduino system. The schematic representation was presented in figure 4.

The basic elements of the platform are a microprocessor, Universal Asynchronous Receiver and Transmitter (UART) which enables communication with a computer through USB and Analogue-Digital Converter (ADC) that enables connection of analogue sensors.

The presented measurement system has a simple construction. An ultrasound HC-SR4 sensor was used for measurement of a distance. The sensor ensured a required precision of measurements, but it should be mentioned that devices with a higher sensitivity are available on the market and their application in the system differs considerably from the presented one. The measurement is performed after the button is pressed and the signal with a result of the measurement is transmitted to a computer through USB. The system is supplied with $9 \mathrm{~V}$ battery or a power supplier. 


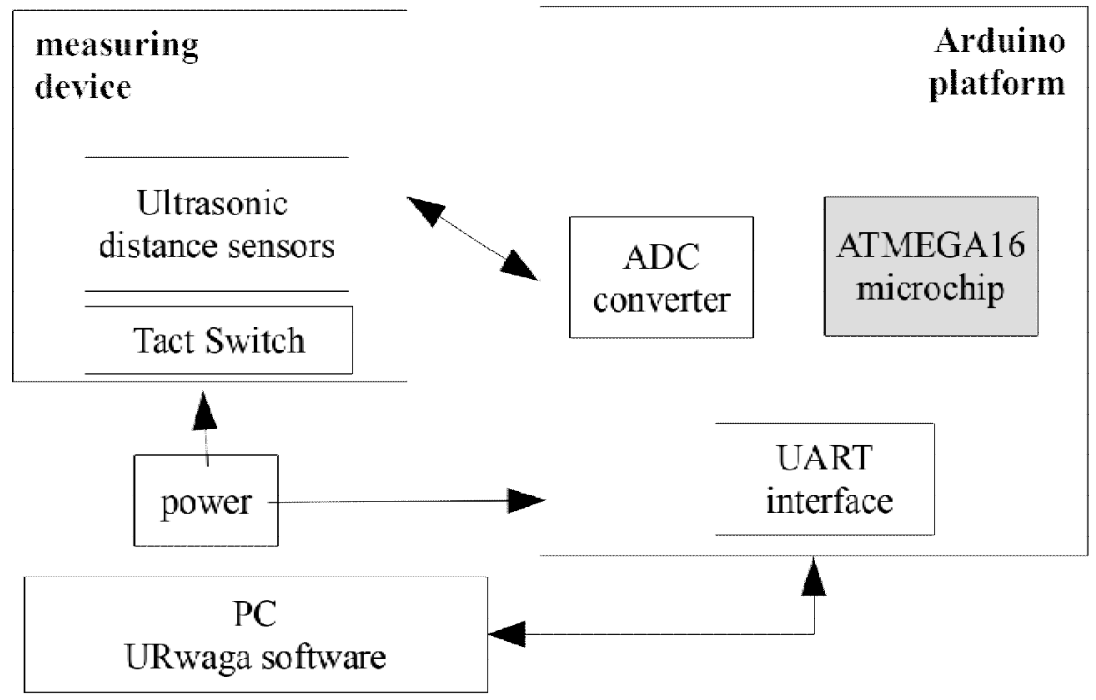

Fig. 4. Schematic representation of the measuring system based on Arduino platform

Before the system is started it is necessary to prepare aforehand a program code. Writing of the program and its recording in the system is enabled $b$ Arduino IDE. A language designed for programming a platform is considerably simple and basic programming skills should be sufficient for its programming. It may be necessary e.g. In case of replacement of the measuring device. Each disconnection of supply causes a need to enter the program code again.

\section{Conclusion}

The presented program was used in the operating measuring system used for studies on agriculture and mechanical engineering. Arduino platform was used in the test stand for measuring of a distance. The system was tested and met the requirements. It should be mentioned that there are many sensors and executive elements which may replace the applied distance sensor [10]. In such a case, after relevant modification of the system and writing of a new program that controls the sensors, measurements of various physical values can be made. In a special case more than one sensor at one time can be used in the system.

URwaga program may be used as a measurement interface for several devices. A possibility of configuration causes that it may be used in various measurement systems. Connection of several devices to the program causes the measurements may be carried out faster that with previously applied methods. The program enabled also to avoid errors while grouping data.

\section{References}

1. J. Axelson, USB Complete: The Developer's Guide (Lakeview research LLC, 2015)

2. M. Margolis, Arduino Cookbook: Recipes to Begin, Expand, and Enhance Your Projects (O'Reilly Media, Inc., 2011)

3. N. M. Z. Hashim, S. R. Mazlan, M. Z. A. Abd Aziz, A. Salleh, A. S. Ja'afar, and N. R. Mohamad, J. Teknol. 77, (2015)

4. D. D. Tung and N. M. Khoa, Eng. Technol. Appl. Sci. Res. 9, 4255 (2019) 
5. A. Szelag-Sikora, M. Niemiec, and J. Sikora, J. Elem. 21, 915 (2016)

6. J. Sikora, M. Niemiec, A. Szelag-Sikora, M. Kubon, E. Olech, and A. Marczuk, Przemysl Chem. 96, 2275-2278 (2017)

7. M. Cupial, A. Szelag-Sikora, J. Sikora, J. Rorat, and M. Niemiec, Ekon. Prawo-Econ. Law 17, 5 (2018)

8. S. Kocira, M. Kuboń, U. Malaga-Toboła, D. Kwaśniewski. Academic Teachers' preferences in Selecting Scientific Databases. Proceedings of the International Scientific Conference, Volume V (2019).

9. A. E. Latawiec, J. B. Krolczyk, M. Kubon, K. Szwedziak, A. Drosik, E. Polanczyk, K. Grotkiewicz, and B. B. N. Strassburg, Sustainability 9, (2017)

10. J. Gonzalez-Buesa and M. L. Salvador, Comput. Electron. Agric. 162, 14 (2019) 